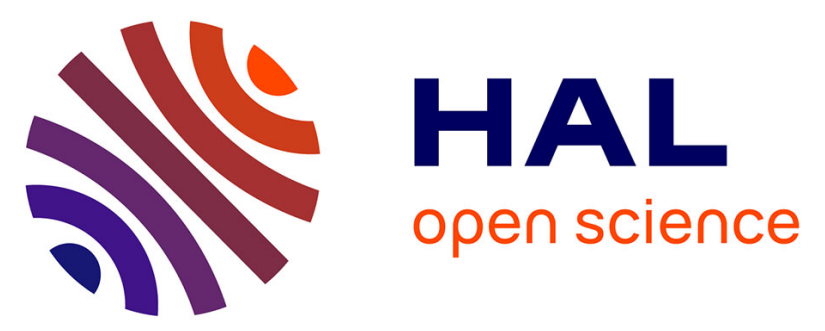

\title{
Observation de transitions de la molécule 29SiH4 dans le spectre Raman stimulé de la bande $\nu 1$ du silane en abondance naturelle
}

\author{
B. Lavorel, R. Saint-Loup, G. Pierre, H. Berger
}

\section{To cite this version:}

B. Lavorel, R. Saint-Loup, G. Pierre, H. Berger. Observation de transitions de la molécule 29SiH4 dans le spectre Raman stimulé de la bande $\nu 1$ du silane en abondance naturelle. Journal de Physique Lettres, 1984, 45 (7), pp.295-300. 10.1051/jphyslet:01984004507029500 . jpa-00232346

HAL Id: jpa-00232346

https://hal.science/jpa-00232346

Submitted on 1 Jan 1984

HAL is a multi-disciplinary open access archive for the deposit and dissemination of scientific research documents, whether they are published or not. The documents may come from teaching and research institutions in France or abroad, or from public or private research centers.
L'archive ouverte pluridisciplinaire HAL, est destinée au dépôt et à la diffusion de documents scientifiques de niveau recherche, publiés ou non, émanant des établissements d'enseignement et de recherche français ou étrangers, des laboratoires publics ou privés. 


\title{
Observation de transitions de la molécule ${ }^{29} \mathrm{SiH}_{4}$ dans le spectre Raman stimulé de la bande $v_{1}$ du silane en abondance naturelle
}

\author{
B. Lavorel, R. Saint-Loup, G. Pierre et H. Berger \\ Laboratoire de Spectronomie Moléculaire de l'Université de Dijon (*), \\ 6, boulevard Gabriel, 21100 Dijon, France
}

(Reçu le 12 janvier 1984, accepté le 6 février 1984)

\begin{abstract}
Résumé. - Le spectre Raman stimulé du silane a été enregistré entre 2183 et $2187 \mathrm{~cm}^{-1}$ sur le spectromètre Raman de Dijon, dont la résolution instrumentale est de $0,0025 \mathrm{~cm}^{-1}$. La résolution du spéctre essentiellement limitée par l'effet Doppler est de $0,006 \mathrm{~cm}^{-1}$ à une pression de 7,5 torr. Cette étude a permis de mettre en évidence des transitions appartenant à l'espèce isotopique ${ }^{29} \mathrm{SiH}_{4}$ et de mesurer le déplacement isotopique entre les centres de la bande $v_{1}$ pour les espèces ${ }^{28} \mathrm{SiH}_{4}$ et ${ }^{29} \mathrm{SiH}_{4}$.

Abstract. - The stimulated Raman spectrum of silane has been recorded between 2183 and $2187 \mathrm{~cm}^{-1}$ with the Raman spectrometer of Dijon which has an instrumental resolution of $0.0025 \mathrm{~cm}^{-1}$. The resolution of the spectrum, essentially limited by the Doppler broadening, is about $0.006 \mathrm{~cm}^{-1}$ at 7.5 torr pressure. In this study we assigned transitions which belong to ${ }^{29} \mathrm{SiH}_{4}$ and we measured the isotopic shift of $v_{1}$ band origins for isotopic species ${ }^{28} \mathrm{SiH}_{4}$ and ${ }^{29} \mathrm{SiH}_{4}$.
\end{abstract}

\section{Introduction.}

Plusieurs études ont déjà été consacrées à la molécule de silane ${ }^{28} \mathrm{SiH}_{4}$. L'état vibrationnel de base $[1,2]$ et les états excités correspondant aux bandes $v_{1}, v_{2}, v_{3}, v_{4}$ ont été étudiés en spectroscopie infrarouge [3, 5] et Raman [5, 6]. Par contre, les espèces isotopiques ${ }^{29} \mathrm{SiH}_{4}$ et ${ }^{30} \mathrm{SiH}_{4}$ dont les abondances naturelles sont 4,70\% et 3,09\% n'ont pas été étudiées jusqu'alors. Nous présentons dans cette lettre le spectre Raman du silane en abondance naturelle obtenu par spectroscopie Raman inverse. Nous avons mis en évidence dans ce spectre des transitions appartenant à l'espèce isotopique ${ }^{29} \mathrm{SiH}_{4}$. Les fréquences des raies intenses de la molécule ${ }^{28} \mathrm{SiH}_{4}$ ont été mesurées en Raman par Owyoung et al. [6]. Ces raies sont utilisées ici pour réaliser l'étalonnage du spectre de l'espèce ${ }^{29} \mathrm{SiH}_{4}$.

\section{Dispositif expérimental.}

Cette expérience a été réalisée à partir d'un processus de diffusion Raman stimulée. Le spectromètre décrit dans une publication antérieure [7] a été modifié pour en accroitre la sensibilité.

Dans une expérience de diffusion Raman stimulée (Fig. 1), on peut observer une amplification de l'onde de fréquence $\omega_{2}$ ou une atténuation de l'onde de fréquence $\omega_{1}$; ce choix dépend de la

(*) Equipe de recherche associée au CNRS, no 599. 


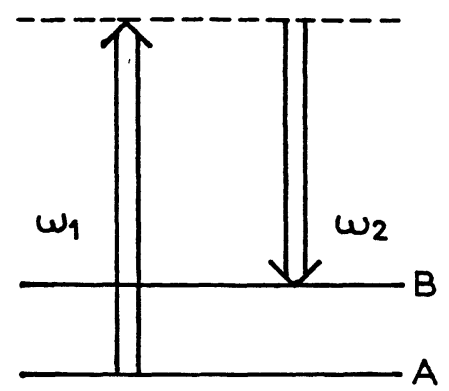

Fig. 1. - Processus Raman stimulé.

[Stimulated Raman process.]

puissánce relative des deux lasers. Dans notre expérience, le laser à la fréquence $\omega_{2}$ est le laser le plus puissant, nous utilisons donc le laser de fréquence $\omega_{1}$ comme "sonde ", mesurant ainsi l'atténuation engendrée par le processus de diffusion. Dans une telle configuration, le phénomène est désigné sous le terme de "Spectroscopie Raman Inverse ». Le rapport signal sur bruit est alors proportionnel à la puissance du laser $\omega_{2}$; c'est pourquoi nous nous sommes tournés vers la réalisation d'une source impulsionnelle de grande monochromaticité.

Réaliser une source accordable puissante et monochromatique est possible en amplifiant en ondes progressives un laser continu de grande finesse spectrale. Un laser à colorant d'environ $100 \mathrm{~mW}$ traverse un système amplificateur constitué de quatre cellules à colorant pompées transversalement par un laser Nd-YAG doublé en fréquence $(\lambda=532 \mathrm{~nm})$. Nous obtenons ainsi une source impulsionnelle de fréquence variable de grande monochromaticité $(\Delta \omega \simeq$ $0,0025^{\circ} \mathrm{cm}^{-1}$ ) et de grande puissance (1 à $3 \mathrm{MW}$ crête).

L'ensemble du dispositif est schématisé sur la figure 2 , les deux ondes $\omega_{1}$ et $\omega_{2}$ ne sont pas coli-

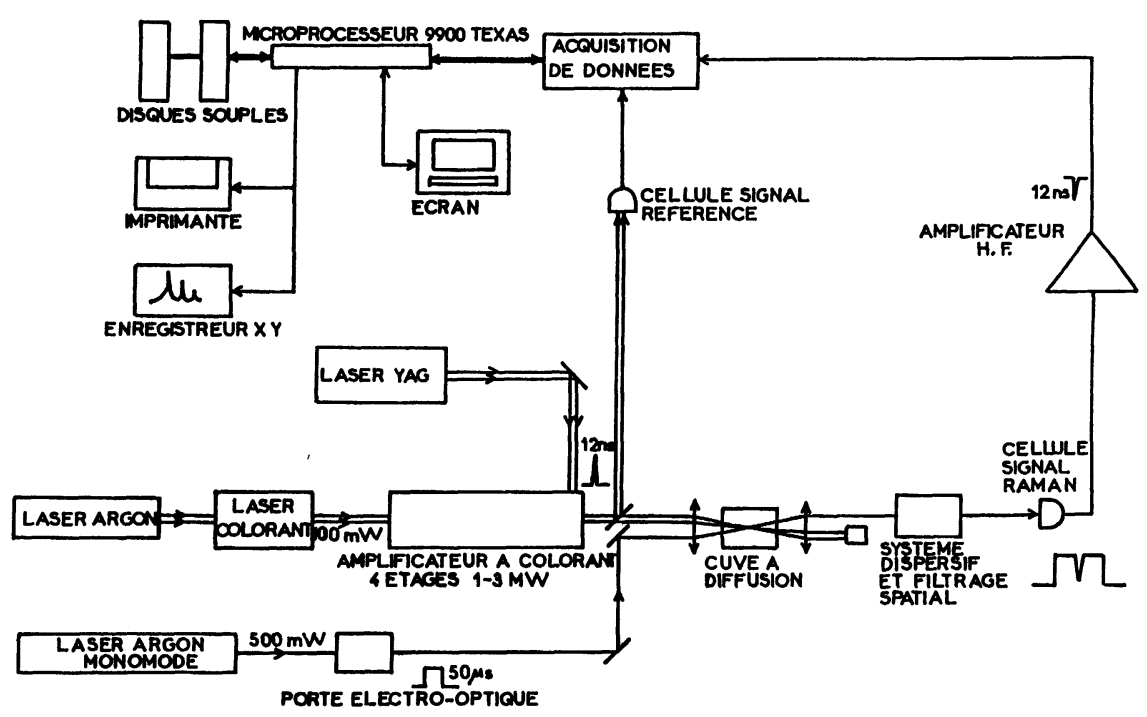

Fig. 2. - Schéma du spectromètre Raman stimulé.

[Schematic diagram of the stimulated Raman spectrometer.] 
néaires, mais croisées dans la cuve à diffusion de façon à rendre plus facile la séparation des faisceaux après interaction et à éviter la création de signaux Raman " parasites » dans les éléments de l'optique, notamment dans les fenêtres de la cuve.

Un système dispersif constitué de deux prismes de Pellin-Brocca et d'un réseau, suivi d'un filtrage spatial, permet d'isoler le signal à la fréquence $\omega_{1}$. Un modulateur électro-optique ne transmet le faisceau sonde sur le détecteur que pendant des intervalles de $50 \mu \mathrm{s}$, ce qui permet de réduire la puissance moyenne sur le détecteur et d'éviter ainsi le phénomène de saturation de la photodiode. Un filtre passe-haut isole le " pic d'absorption " correspondant à l'atténuation de l'onde $\omega_{1}$.

Le signal, mesuré grâce à un échantillonneur bloqueur rapide, est moyenné dans un système d'acquisition de données. Enfin, les données sont stockées sur disquette.

\section{Résultats et discussion.}

Le spectre observé, constitué de plusieurs enregistrements d'un $\mathrm{cm}^{-1}$ environ, est représenté sur la figure $3 a$. La résolution obtenue est de $0,006 \mathrm{~cm}^{-1}$ à une pression de 7,5 torr et une température de $294 \mathrm{~K}$. Les raies les plus intenses du spectre correspondent aux transitions permises de la branche $Q$ de la molécule ${ }^{28} \mathrm{SiH}_{4}$.

Un spectre calculé a été obtenu à partir de l'analyse des données infrarouges enregistrées sur le spectromètre à transformée de Fourier du Laboratoire d'Infrarouge d'Orsay [9]. Cette analyse porte sur les trois espèces isotopiques du silane et a permis de prévoir les spectres calculés des figures $3 b, 3 c$ et $3 d$.

En comparant le spectre expérimental et le spectre calculé de ${ }^{28} \mathrm{SiH}_{4}$ (Figs. $3 a$ et $3 b$ ), on peut voir qu'un certain nombre de raies faibles numérotées sur le spectre expérimental, n'appartiennent pas à la bande $v_{1}$ de ${ }^{28} \mathrm{SiH}_{4}$.

Ces transitions correspondent à la branche $Q$ de l'espèce isotopique ${ }^{29} \mathrm{SiH}_{4}$. Le spectre calculé de la molécule ${ }^{29} \mathrm{SiH}_{4}$, déduit de l'analyse infrarouge [9] est en bon accord avec le spectre expérimental.

$A$ priori, on aurait pu s'attendre à observer de la même façon la branche $Q$ de l'isotope ${ }^{30} \mathrm{SiH}_{4}$; mais, comme le montre le spectre calculé (Fig. $3 d$ ), les raies du ${ }^{30} \mathrm{SiH}_{4}$ sont nettement plus faibles que celles $\mathrm{du}^{29} \mathrm{SiH}_{4}$. Il y a deux raisons à ce phénomène :

- La proportion de cette espèce est légèrement plus faible $\left(4,7 \%\right.$ pour ${ }^{29} \mathrm{SiH}_{4}$ et $3,1 \%$ pour $\left.{ }^{30} \mathrm{SiH}_{4}\right)$.

- L'hybridation entre les niveaux $v_{1}=1$ et $v_{3}=1$ essentiellement due au déplacement isotopique du centre de bande de $v_{3}$ est plus important pour cet isotope. Il en résulte un éclatement tétraédrique plus important, supprimant le recouvrement de raies dès les faibles valeurs de $J$ et un respect moins rigoureux de la règle de sélection $\Delta R$ et $\Delta n=0$ qui a pour effet de distribuer l'intensité sur un plus grand nombre de transitions.

En général le spectre d'absorption de l'iode [8], enregistré simultanément, permet d'effectuer l'étalonnage des spectres Raman. Ici, nous avons utilisé comme étalons les fréquences des sommets des raies Raman pointés sur le spectre calculé. Pour réaliser le tracé du spectre calculé, le profil de raie utilisé est un profil de Voigt, résultant des élargissements collisionnels $\left(0,003 \mathrm{~cm}^{-1}\right.$ de largeur à mi-hauteur) et Doppler $\left(0,0047 \mathrm{~cm}^{-1}\right)$ et de la fonction d'appareil assimilée à une fonction Gaussienne $\left(0,0025 \mathrm{~cm}^{-1}\right)$.

Nous avons préféré comme étalon le spectre calculé de ${ }^{28} \mathrm{SiH}_{4}$ au spectre expérimental [6] car ce dernier n'était pas enregistré dans les mêmes conditions de résolution et de pression que notre spectre, ce qui peut modifier la position du sommet des raies. D'autre part, les données expérimentales du spectre infrarouge du silane sont très précises et l'analyse reproduit ces données avec une précision de l'ordre de $0,0005 \mathrm{~cm}^{-1}$ [9].

Les fréquences et les attributions des raies observées de ${ }^{29} \mathrm{SiH}_{4}$ sont indiquées dans le tableau I. 


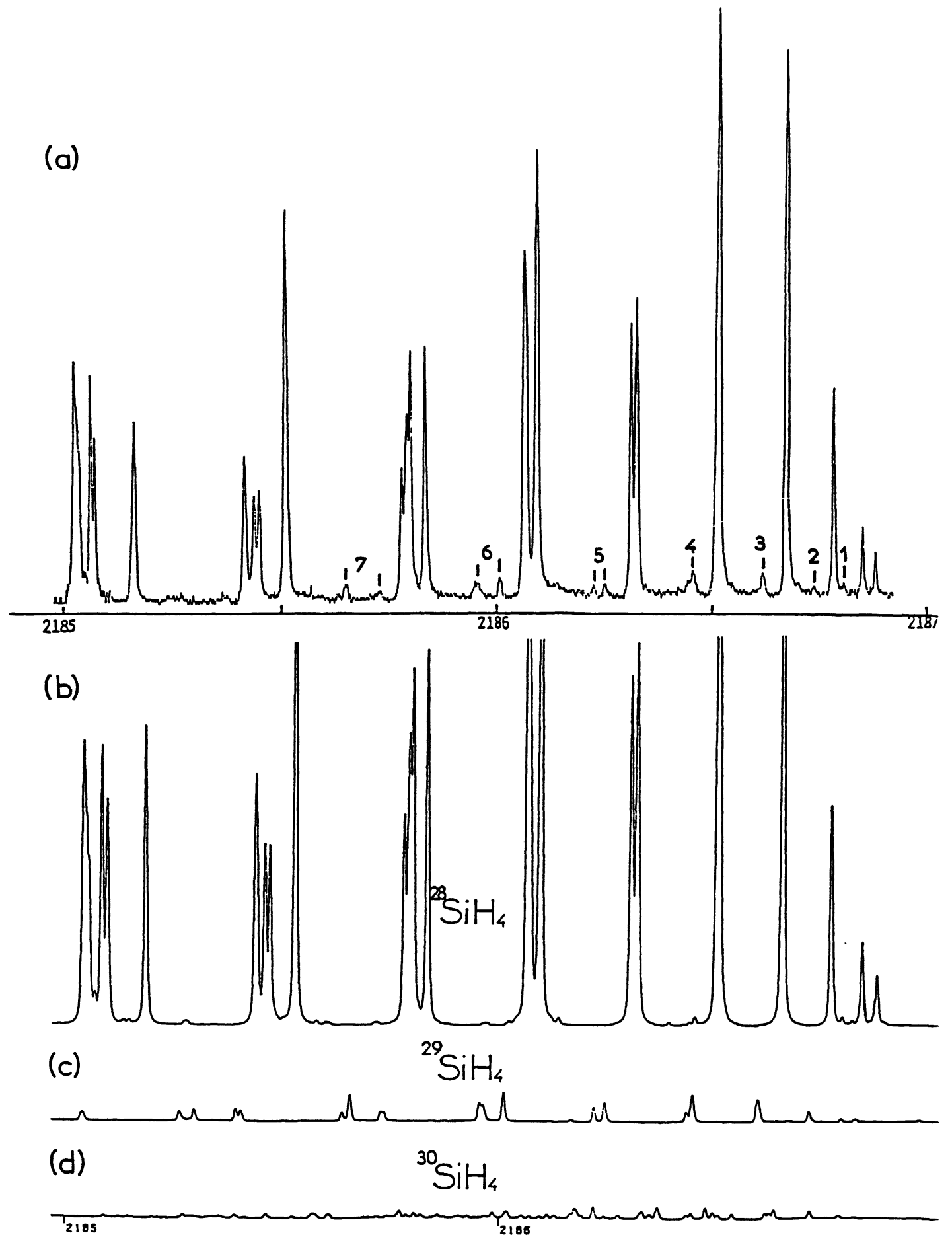

Fig. 3. - Spectres Raman du silane : $a$ ) experimental (abondance naturelle); $b$ ), $c$ ), $d$ ) spectres calculés des espèces ${ }^{28} \mathrm{SiH}_{4},{ }^{29} \mathrm{SiH}_{4}$ et ${ }^{30} \mathrm{SiH}_{4}$.

[Raman spectra of silane : a) experimental (natural silane); $b$ ), c), d) synthetic spectra of ${ }^{28} \mathrm{SiH}_{4},{ }^{29} \mathrm{SiH}_{4}$ and $\left.{ }^{30} \mathrm{SiH}_{4} \cdot\right]$ 
Tableau I. - Transitions Raman de ${ }^{29} \mathrm{SiH}_{4}$.

[Raman transitions of ${ }^{29} \mathrm{SiH}_{4}$.]

\begin{tabular}{|c|c|c|c|c|}
\hline ATTRIBUTION & $\begin{array}{l}\text { FREQUENCE } \\
\text { OBSERVEE } \\
\left(\mathrm{cm}^{-1}\right)\end{array}$ & $\begin{array}{l}\text { FREQUENCE } \\
\text { CALCULEE [*] } \\
\left(\mathrm{cm}^{-1}\right)\end{array}$ & $\begin{array}{c}\text { OBS - CALC. } \\
\left(\mathrm{cm}^{-1}\right)\end{array}$ & $\begin{array}{l}\text { CENTRE DE STRUCTURE } \\
\left(\mathrm{cm}^{-1}\right)\end{array}$ \\
\hline$a_{1} \quad F_{1}$ & 2186.7893 & 2186.7925 & -.0032 & 2186.7893 \\
\hline $\begin{array}{ll} & E \\
Q_{2} & F_{2}\end{array}$ & 2186.7177 & 2186.7197 & -.0020 & 2186.7177 \\
\hline$Q_{3} \begin{array}{l}A_{2} \\
F_{1} \\
F_{2}\end{array}$ & 2186.6014 & 2186.6035 & -.0021 & 2186.6014 \\
\hline $\begin{array}{ll} & A_{1} \\
& E \\
Q_{4} & F_{1} \\
& F_{2}\end{array}$ & 2186.4540 & 2186.4531 & +.0009 & 2186.4540 \\
\hline $\begin{array}{ll} & F_{1} \\
Q_{5} & F_{2}\end{array}$ & 2186.2550 & 2186.2539 & +.0011 & 21862431 \\
\hline $\begin{array}{ll}a_{5} & E \\
& F_{1}^{\prime}\end{array}$ & 2186.2302 & 2186.2285 & +.0017 & \\
\hline $\begin{array}{ll}a_{6} & A_{2} \\
& F_{2}^{\prime}\end{array}$ & 2186.0122 & 2186.0132 & -.0010 & 2959 9977 \\
\hline$a_{6} \begin{array}{l}A_{1} \\
F_{1} \\
F_{2}\end{array}$ & 2185.9580 & 2185.9628 & -.0048 & \\
\hline $\begin{array}{l}Q_{7} \\
F_{1} \\
F_{2}\end{array}$ & 2185.7280 & 2185.7280 & +.0000 & \\
\hline $\begin{array}{ll} & \mathrm{A}_{2} \\
& E^{2} \\
a_{7} & F_{1}^{\prime} \\
& F_{2}^{\prime}\end{array}$ & 2185.6589 & 2185.6567 & +.0022 & 2185.6817 \\
\hline
\end{tabular}

[*] : Fréquence du sommet pointé sur le spectre calculé de ${ }^{29} \mathrm{SiH}_{4} \cdot$ [9]

Les fréquences des raies observées ont été mesurées puis comparées aux fréquences des sommets correspondants pointés sur le spectre calculé [9]. L'écart type sur l'ensemble de ces données est de $0,0023 \mathrm{~cm}^{-1}$.

Nous avons ensuite déterminé pour chaque valeur de $J$ le centre de la structure tétraédrique (Tableau I) et en utilisant une régression polynomiale de degré deux en $J(J+1)$, nous en avons déduit les paramètres suivants :

$$
\begin{aligned}
& \alpha_{1}-\alpha_{0}=2186,827(3) \mathrm{cm}^{-1} \\
& \beta_{1}-\beta_{0}=-18,2(3) \times 10^{-3} \mathrm{~cm}^{-1} \\
& \gamma_{1}-\gamma_{0}=-4,2(5) \times 10^{-5} \mathrm{~cm}^{-1}
\end{aligned}
$$

Ces paramètres correspondent à une étude de la bande $v_{1}$ considérée sans interaction explicite avec la bande $v_{3}$, donc différent notablement de ceux obtenus dans la référence [9]. 
Le déplacement isotopique entre les espèces ${ }^{28} \mathrm{SiH}_{4}$ et ${ }^{29} \mathrm{SiH}_{4}$ est de $-0,045 \mathrm{~cm}^{-1}$. En utilisant une relation linéaire entre $v_{1}$ et $\left(m_{\mathrm{Si}}\right)^{-1 / 2}$ on prévoit le centre de bande du $3 \mathrm{e}$ isotope $: v_{1}\left({ }^{30} \mathrm{SiH}_{4}\right)=$ $2186,784 \mathrm{~cm}^{-1}$, soit un déplacement isotopique de $-0,088 \mathrm{~cm}^{-1}$ entre ${ }^{28} \mathrm{SiH}_{4}$ et ${ }^{30} \mathrm{SiH}_{4}$.

En conclusion, à partir du spectre Raman stimulé du silane en abondance naturelle, nous avons identifié et mesuré la fréquence des raies observées de la bande $v_{1}$ de ${ }^{29} \mathrm{SiH}_{4}$. La précision sur les mesures est de $0,003 \mathrm{~cm}^{-1}$. A partir de ces données, nous avons déterminé le déplacement isotopique par rapport à l'isotope principal, du centre de la bande $v_{1}$ de ${ }^{29} \mathrm{SiH}_{4}$ et prévu celui de la molécule ${ }^{30} \mathrm{SiH}_{4}$.

\section{Remerciements.}

Les auteurs remercient $\mathbf{R}$. Chaux et $\mathrm{Ch}$. Wenger pour leur participation à la réalisation de l'électronique du dispositif expérimental [10].

Ce travail a bénéficié du soutien financier de la Direction des Recherches, Etudes et Techniques (Contrat 81-132).

\section{Bibliographie}

[1] Ozier, I., Lees, R. M. et Gerry, M. C. L., Can. J. Phys. 54 (1976) 1094-1105.

[2] Pierre, G., Valentin, A., Henry, L., Can. J. Phys. (à paraître).

[3] Cabana, A., Gray, D. L., Robiette, A. G. et Pierre, G., Mol. Phys. 36 (1978) 1503-1516.

[4] Gray, D. L., Robiette, A. G. et Johns, J. W. C., Mol. Phys. 34 (1977) 1437-1453.

[5] Pierre, G., Champion, J. P., Kozlov, D. N. et Smirnov, V. V., J. Physique 43 (1982) 1429-1436.

[6] Owyoung, A., Eshrick, P., Robiette, A. G. et McDowell, R. S., J. Mol. Spectrosc. 86 (1981) 209-215.

[7] Mao, S. Q., Saint-Loup, R., Aboumajd, A., Lepage, P., Berger, H. et Robiette, A. G., J. Raman Spectrosc. 13 (1982) 257-261.

[8] Gerstenkorn, S., Luc, P., Atlas du spectre d'absorption de la molécule d'iode $14800-20000 \mathrm{~cm}^{-1}$ (Editions du C.N.R.S.).

[9] Lepage, P., Bouzouba, K., Pierre, G. et Guelachvili, G., Colloque de Spectronomie Moléculaire de Tours (1983) papier en préparation.

[10] Chaux, R. et Wenger, Ch., Micro-bulletin du C.N.R.S. (à paraître). 